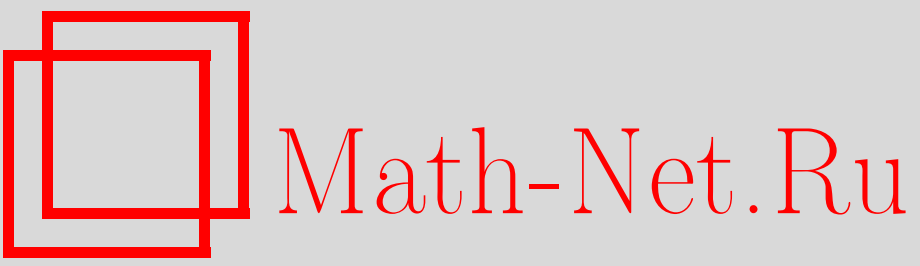

А. А. Макаров, Краевая задача для систем сверточных уравнений в анизотропных пространствах функций, Итоги науки и техн. Сер. Соврем. мат. и ее прил. Темат. обз., 2020, том 182, 66-69

DOI: https://doi.org/10.36535/0233-6723-2020-182-66-69

Использование Общероссийского математического портала Math-Net.Ru подразумевает, что вы прочитали и согласны с пользовательским соглашением

http://www. mathnet.ru/rus/agreement

Параметры загрузки:

IP : 54.147 .182 .235

26 апреля 2023 г., $06: 24: 46$ 


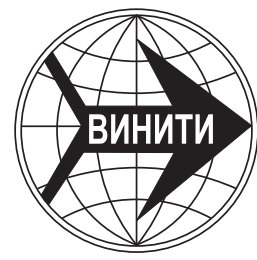

ИТОГИ НАУКИ И ТЕХНИКИ.

Современная математика и ее приложения.

Тематические обзоры.

Том 182 (2020). С. 66-69

DOI: $10.36535 / 0233-6723-2020-182-66-69$

УДК 517.954

\title{
КРАЕВАЯ ЗАДАЧА ДЛЯ СИСТЕМ СВЕРТОЧНЫХ УРАВНЕНИЙ В АНИЗОТРОПНЫХ ПРОСТРАНСТВАХ ФУНКЦИЙ
}

\author{
(c) 2020 г. A. A. MAKAPOB
}

\begin{abstract}
АннотАция. В работе получены анизотропные классы корректности задачи Коши и краевой задачи для систем уравнений в свертках. Для частного случая дифференциальных уравнений используется гиперповерхность сопряженных порядков соответствующего многочлена и с ее помощью получаются различные классы корректности.
\end{abstract}

Ключевъе слова: уравнение в свертках, краевая задача, преобразование Фурье, гиперповерхность сопряженных порядков, анизотропное пространство функций.

\section{BOUNDARY-VALUE PROBLEM FOR SYSTEMS OF CONVOLUTIONAL EQUATIONS IN ANISOTROPIC FUNCTIONAL SPACES}

\author{
(c) 2020 A. A. MAKAROV
}

\begin{abstract}
In this paper, anisotropic classes of well-posed Cauchy problems and boundary-value problems for systems of convolutions equations are obtained. For a particular case of differential equations, a hypersurface of conjugate orders of the corresponding polynomial is used, and various classes of well-posed problems are obtained.
\end{abstract}

Keywords and phrases: convolution equation, boundary-value problem, Fourier transform, hypersurface of conjugate orders, anisotropic space of functions.

AMS Subject Classification: 35A05, 35S10

Задача Коши для дифференциальных уравнений в частных производных рассматривается, как правило, в изотропных пространствах, т.е. когда по разным пространственным переменным рост решения одинаковый. Лишь в работах Л. Р. Волевича и С. Г. Гиндикина $[1,2]$ рассматривались уравнения в свертках в пространствах функций экспоненциального роста, в которых различным переменным соответствовал различный рост. И только в работе Г. П. Сердюка [5] были получены анизотропные классы единственности решений задачи Коши для систем дифференциальных уравнений.

В данной работе получены анизотропные классы корректности задачи Коши и краевой задачи для систем уравнений в свертках, обобщающих дифференциальные и дифференциальноразностные уравнения.

Рассматривается краевая задача

$$
\begin{gathered}
2 \frac{\partial u(x, t)}{\partial t}=A(x) * u(x, t), \quad x \in \mathbb{R}^{n}, \quad t \in[0, T], \\
B(x) * u(x, 0)+C(x) * u(x, T)=\varphi(x),
\end{gathered}
$$


где $u(x, t), \varphi(x)$ - вектор-функции, принадлежащие некоторому линейному топологическому пространству $\Phi$ при всех $t \in[0, T] ; A(x), B(x)$ и $C(x)$ - матрицы, компоненты которых являются свертывателями в пространстве $\Phi$.

Задача (1)-(2) называется корректной в пространстве $\Phi$, если для любой функции $\varphi(x) \in \Phi$ существует единственное решение $u(x, t) \in \Phi, t \in[0, T]$, задачи (1)-(2) и это решение непрерывно зависит от $\varphi(x)$ в топологии пространства $\Phi$.

При исследовании корректности краевой задачи (1)-(2) важную роль играет разрешающая матрица этой задачи

$$
Q(s, t)=\exp t \widetilde{A}(s)(\widetilde{B}(s)+\widetilde{C}(s) \exp T \widetilde{A}(s))^{-1},
$$

где $\widetilde{A}(s), \widetilde{B}(s)$ и $\widetilde{C}(s)$ - преобразования Фурье матриц $A(x), B(x)$ и $C(x)$. В [3,4] автором был доказан критерий корректности краевой задачи (1)-(2).

Теорема 1. Пусть пространство $\Phi$ является классом единственности задачи Коши для системы (1). Краевая задача (1)-(2) корректна в пространстве $\Phi$ тогда и только тогда, когда $Q(s, t) \in F C(\Phi)$, т.е. является преобразованием Фуръе от свертывателя в пространстве $\Phi$.

В качестве пространства $\Phi$ будем рассматривать проективный предел банаховых пространств

$$
W_{L, \alpha}^{s}=\left\{\varphi(x) \in C^{s}\left(\mathbb{R}^{n}\right):\|\varphi\|=\sup _{x \in \mathbb{R}^{n},|k| \leqslant s}\left|D^{k} \varphi(x)\right| \exp \left(-\sum_{j=1}^{n} l_{j}\left(\alpha_{j}|x|\right)\right)<\infty\right\},
$$

где $\alpha_{j}>0$, а $l_{j}(\xi)$ - выпуклая непрерывная положительная функция, растущая быстрее любой линейной функции. Тогда

с топологией проективного предела.

$$
W_{L}^{\infty}=\bigcap_{s \in \mathbb{N}, \alpha>0} W_{L, \alpha}^{s}
$$

В [4] показано, что преобразования Фурье свертывателей этого пространства принадлежат пространству

$$
\Omega_{L^{*}}=\left\{\psi(s) \text { - целая функция: }|\psi(s)| \leqslant C_{0}(1+|s|)^{p} \exp \sum_{j=1}^{n} l_{j}^{*}\left(\beta_{j}\left|\operatorname{Im} s_{j}\right|\right)\right\},
$$

где $l_{j}^{*}(\eta)$ - двойственная по Юнгу к $l_{j}(\xi)$ с некоторым $\beta_{j}>0$.

Будем также использовать следующие пространства из [1]:

$$
S_{[\nu]}=\bigcap_{s, l} C_{l[\nu]}^{s},
$$

где $C_{l[\nu]}^{s}$ - банахово пространство $s$ раз непрерывно дифференцируемых функций с нормой

$$
\|\varphi\|=\sup _{x \in \mathbb{R}^{n},|\alpha| \in s} \exp \left(-\sum_{j=1}^{n} \nu_{j}\left|x_{j}\right|\right)\left(1+|x|^{2}\right)^{l / 2}\left|D^{\alpha} \varphi(x)\right| .
$$

В [1] показано, что преобразование Фурье свертывателя принадлежит пространству

$$
\Psi_{[\nu]}=\left\{\psi(s) \text { - голоморфная в слое }\left|\operatorname{Im} s_{j}\right|<\nu_{j},|\varphi(s)|<c(1+|s|)^{p} d\left(\operatorname{Im} s_{j}\right)^{-h}\right\},
$$

где $d\left(\operatorname{Im} s_{j}\right)$ - расстояние от $s$ до границы слоя.

Применяя теорему 1 к введенным пространствам, получим следующие результаты.

Теорема 2. Краевая задача (1)-(2) корректна в пространстве $W_{L}^{\infty}$ тогда и только тогда, когда $Q(s, t) \in \Omega_{L^{*}}$.

Теорема 3. Краевая задача (1)-(2) корректна в пространстве $S_{[\nu]}$ тогда и только тогда, когда $Q(s, t) \in \Psi_{[\nu]}$.

Замечание 1. Можно рассмотреть также функциональные пространства, в которых по одним параметрам функция растет как $\exp b\left|x_{k}\right|^{p_{k}}$, а по другим - как $\exp \nu_{k}\left|x_{k}\right|$. 
Замечание 2. Так как разрешающий оператор является сверткой в заданном пространстве, то его можно продолжить на функции конечной гладкости и получить корректную разрешимость краевой задачи в шкале соответствующих банаховых пространств.

В качестве примера рассмотрим краевую задачу

$$
\begin{gathered}
\frac{\partial u\left(x_{1}, x_{2}, t\right)}{\partial t}=a \frac{\partial^{2} u}{\partial x_{1}^{2}}+b \frac{\partial u}{\partial x_{2}}, \quad a, b \neq 0, \\
u\left(x_{1}, x_{2}, 0\right)+u\left(x_{1}, x_{2}-b T, T\right)=\varphi(x) .
\end{gathered}
$$

Здесь $\widetilde{A}(s)=-a s_{1}^{2}+b i s_{2}, \widetilde{B}(s)=1, \widetilde{C}(s)=\exp ^{-i b s_{2} T}$. Тогда

$$
Q\left(s_{1}, s_{2}, t\right)=\frac{\exp t\left(-a s_{1}^{2}+i b s_{2}\right)}{\left(1+\exp \left(-T a s_{1}^{2}\right)\right)}
$$

Знаменатель $\left.1+\exp \left(-T a s_{1}^{2}\right)\right)$ не обращается в нуль при $\left|\operatorname{Im} s_{1}\right|<\sqrt{\pi /(T|a|)}=\nu_{1}$. Значит, в слое $\left|\operatorname{Im} s_{1}\right|<\sqrt{\pi /(T|a|)}, s_{2} \in \mathbb{C}$ выполняется неравенство

$$
\left|Q\left(s_{1}, s_{2}, t\right)\right| \leqslant C \exp |b| \cdot\left|\operatorname{Im} s_{2}\right| \cdot d^{-1}\left(\operatorname{Im} s_{1}\right) .
$$

Следовательно, данная краевая задача корректна в пространстве бесконечно дифференцируемых функций, удовлетворяющих оценке

$$
\left|D^{k} \varphi(x)\right| \leqslant C \exp \left(l\left(\alpha\left|x_{2}\right|\right)+\beta\left|x_{1}\right|\right),
$$

где $\beta \in\left(0, \nu_{1}\right)$, a $l\left(\left|x_{2}\right|\right)$ - любая выпуклая положительная функция, растущая быстрее линейных функций.

Рассмотрим теперь частный случай нашей краевой задачи-задачу Коши для дифференциальных уравнений с постоянными коэффициентами:

$$
\frac{\partial u(x, t)}{\partial t}=P\left(\frac{\partial}{\partial x}\right) u(x, t), \quad \text { где } \quad P(i s)=\sum_{|k| \leqslant m} a_{k} s_{1}^{k_{1}} \ldots s_{n}^{k_{n}} .
$$

Для таких уравнений в [5] было введено понятие гиперповерхности сопряженных порядков

$$
S=\left\{\alpha \in \mathbb{R}_{+}^{n}: \max _{k: \alpha_{k} \neq 0} \sum_{i=1}^{n} \frac{k_{i}}{\alpha_{i}} \leqslant 1\right\} .
$$

В той же работе был получен следующий результат: если $\alpha \in S$, причем $\alpha_{1}, \ldots, \alpha_{r}>1$, а $\alpha_{r+1}, \ldots, \alpha_{n}=1$, и решение задачи Коши с нулевым начальным условием удовлетворлет оченке

$$
|u(x, t)| \leqslant C \exp \left(\sum_{k=1}^{r} c_{k}\left|x_{k}\right|^{\alpha_{k}^{\prime}}+\sum_{k=r+1}^{n} c_{k}\left|x_{k}\right|^{b_{k}}\right) \quad \forall t \in[0, T],
$$

где $\alpha_{k}^{\prime}=\alpha_{k} /\left(\alpha_{k}-1\right)$ - сопряженный порядок, а $b_{k}$-любое число, большее 1, то $u(x, t) \equiv 0$. Применяя к задаче Коши теорему 2, получим следующий результат.

Теорема 4. Если полином $Q(\tau, s)=\tau-P($ is $)$ является экспоненииально корректным (см. [2]), m.e. $\operatorname{Re} P(i s)<C(\nu)$ nри $|\operatorname{Im} s|<\nu$ при всех $\nu>0 u \alpha \in S$, причем $\alpha_{j}>1$ для $j=1, r, \ldots u$ $\alpha_{j}=1$ для $j=r+1, n$, то задача Кочи корректна в пространстве $W_{L}^{\infty}$, где $l_{j}\left(\left|x_{j}\right|\right)=\left|x_{j}\right|^{\alpha_{j}^{\prime}}$ nри $j \leqslant r$, a $l_{j}\left(\left|x_{j}\right|\right)=\left|x_{j}\right|^{b_{j}}$ при $j>r$ для всех $b_{j}>1$.

Доказательство. Разрешающая функция $Q(s, t)=\exp t(P(i s))$ удовлетворяет оценке

$$
|Q(s, t)| \leqslant \exp T \operatorname{Re} P(i s) \leqslant C \exp T\left(\sum_{j=1}^{r} c_{j}\left|\operatorname{Im} s_{j}\right|^{\alpha_{j}}+\sum_{j>r} c_{j}\left|\operatorname{Im} s_{j}\right|\right)
$$

(см. [5]), откуда и следует утверждение теоремы. 
Пример. Рассмотрим задачу

$$
\begin{gathered}
\frac{\partial u\left(x_{1}, x_{2}, t\right)}{\partial t}=a \frac{\partial^{2 p} u\left(x_{1}, x_{2}, t\right)}{\partial x_{1}^{2 p}}+c \frac{\partial^{m+n} u\left(x_{1}, x_{2}, t\right)}{\partial x_{1}^{m} \partial x_{2}^{n}}+b \frac{\partial^{2 q} u\left(x_{1}, x_{2}, t\right)}{\partial x_{2}^{2 q}} \\
u\left(x_{1}, x_{2}, 0\right)=\varphi\left(x_{1}, x_{2}\right) .
\end{gathered}
$$

Здесь гиперповерхность сопряженных порядков задается неравенствами

$$
\alpha_{1} \geqslant 2 p, \quad \alpha_{2} \geqslant 2 q, \quad \frac{m}{\alpha_{1}}+\frac{n}{\alpha_{2}} \leqslant 1 .
$$

Пусть $m \leqslant p, n \leqslant q$. Тогда можно взять $\alpha_{1}=2 p, \alpha_{2}=2 q$. Если $(-1)^{p} a<0$ и $(-1)^{q} b<0$, то при достаточно малых $|c|$ данная задача Коши корректна в пространстве $W_{L}^{\infty}$ с функциями $l_{1}\left(\left|x_{1}\right|\right)=\left|x_{1}\right|^{(2 p)^{\prime}}$ и $l_{2}\left(\left|x_{2}\right|\right)=\left|x_{2}\right|^{(2 q)^{\prime}}$.

\section{СПИСОК ЛИТЕРАТУРЫ}

1. Волевич Л. Р., Гиндикин С. Г. Задача Коши и связанные с ней задачи для уравнений типа свертки// Усп. мат. наук. - 1972. - 27, № 4 (166). - С. 65--143.

2. Волевич Л. Р., Гиндикин С. Г. Обобщенные функции и уравнения в свертках. - М.: Физматлит, 1994.

3. Макаров A. A. Критерий корректной разрешимости краевой задачи в слое для системы линейных уравнений в свертках в топологических пространствах// в кн.: Теоретические и прикладные вопросы дифференциальных уравнений и алгебры. - Киев: Наукова думка, 1978. - С. 178-180.

4. Макаров A. А. О необходимых и достаточных условиях корректной разрешимости краевой задачи в слое для систем дифференциальных уравнений в частных производных// Диффер. уравн. - 1981. 17, № 2. - C. 320-324.

5. Сердюк Г. П. О единственности решений линейных дифференциальных уравнений/ Дисс. на соиск. уч. степ. канд. физ.-мат. наук - Харьков: ХГУ, 1983.

Макаров Александр Анатольевич

Харьковский национальный университет им. В. Н. Каразина, Харьков, Украина

E-mail: natvasmak@ukr.net 